\title{
Reduction of inbreeding in commercial females by rotational mating with several sire lines
}

\author{
Takeshi HondA $^{\mathrm{a}}$, Tetsuro NomURA ${ }^{\mathrm{b}}$, Fumio MUKAI ${ }^{\mathrm{c} *}$ \\ ${ }^{a}$ Graduate School of Science and Technology, Kobe University, Kobe, Japan \\ ${ }^{\mathrm{b}}$ Faculty of Engineering, Kyoto Sangyo University, Kyoto, Japan \\ ${ }^{\mathrm{c}}$ Faculty of Agriculture, Kobe University, Kobe, Japan
}

(Received 17 November 2003; accepted 27 April 2004)

\begin{abstract}
A mating system to reduce the inbreeding of commercial females in the lower level was examined theoretically, assuming a hierarchical breed structure, in which favorable genes are accumulated in the upper level by artificial selection and the achieved genetic progress is transferred to the lower level through migration of males. The mating system examined was rotational mating with several closed sire lines in the upper level. Using the group coancestry theory, we derived recurrence equations for the inbreeding coefficient of the commercial females. The asymptotic inbreeding coefficient was also derived. Numerical computations showed that the critical factor for determining the inbreeding is the number of sire lines, and that the size of each sire line has a marginal effect. If four or five sire lines were available, rotational mating was found to be quite an effective system to reduce the short- and long-term inbreeding of the commercial females, irrespective of the effective size of each sire line. Oscillation of the inbreeding coefficient under rotational mating with initially related sire lines could be minimized by avoiding the consecutive use of highly related lines. Extensions and perspectives of the system are discussed in relation to practical application.
\end{abstract}

inbreeding / coancestry / rotational mating / commercial females

\section{INTRODUCTION}

The control of the increase of inbreeding is a common policy in the maintenance of animal populations. To reduce the inbreeding rate in conserved populations or control lines in selection experiments, many strategies, such as equalization of family sizes [10,29], choice of parents to minimize average coancestry [4,27] and various systems of group mating [17, 24, 29] have been proposed.

\footnotetext{
*Corresponding author: mukai@ans.kobe-u.ac.jp
} 
In most animal breeds in commercial use, the solution of the inbreeding problem will be complicated by the hierarchical structure, in which favorable genes are accumulated in the upper level of the hierarchy by artificial selection and the achieved genetic progress is transferred to the lower level mainly through the migration of males $[23,28]$. In such a structure, different systems are required for reducing the inbreeding rate in the breed, according to the levels of the hierarchy. As shown by many authors [16, 21, 25, 31], selection is inevitably accompanied by an increase in inbreeding. Thus, the main problem of inbreeding in the upper level of the hierarchy is to maximize the genetic progress under a restricted increase of inbreeding, and a large number of selection and mating systems for this purpose have been developed (e.g. [5, 15,26]).

In the present study, we focused on a mating system to reduce the inbreeding rate in commercial females in the lower level of the hierarchy. Farmers in the lower level of the hierarchy usually rear females to produce commercial products and their replacements. Since the traits related to commercial production and reproduction can show strong inbreeding depression $[9,20]$, the suppression of increased inbreeding in the commercial females will be a practically important issue. We supposed a situation where males are supplied by several strains (referred to as "sire lines" hereafter) in the upper level of the hierarchy. One of the most efficient systems will be the rotational use of the sire lines, as in rotational crossing with several breeds. The use of this mating system to reduce the inbreeding in commercial females was first proposed by Nozawa [19]. Using the methodology of path analysis, he worked out the recurrence equation of the inbreeding coefficient under rotational mating with full-sib mated sire lines, and showed that this type of mating is quite an effective system to reduce the long-term inbreeding accumulation in commercial females [19]. In this study, we derive more general recurrence equations, which allow the evaluation of the effects of the number and size of sire lines and the initial relationship among them. Based on numerical computations with the equations obtained, the practical efficiency of the rotational mating system was examined.

\section{MODELS AND ASSUMPTIONS}

\subsection{Theory of group coancestry}

In the derivation, we applied the group coancestry theory $[6,7]$, which is an extension of the coancestry of individuals [14] to groups of individuals. Under random mating, the group coancestry has the same operational rule 
as the ordinary coancestry. For example, consider the group of individuals $x$ with parental groups of $p$ and $q$, each of which descended from grandparental groups of $a, b$, and $c, d$, respectively. Letting $\phi_{p \cdot q}$ be the group coancestry between two groups $p$ and $q$, the expected inbreeding coefficient $\left(F_{x}\right)$ of individuals in group $x$ is expressed as:

$$
F_{x}=\phi_{p \cdot q}=\frac{1}{4}\left[\phi_{a \cdot c}+\phi_{a \cdot d}+\phi_{b \cdot c}+\phi_{b \cdot d}\right] .
$$

The group coancestry of group $x$ with itself is defined as the average pairwise coancestry including reciprocals and self-coancestries [4]. Thus,

$$
\phi_{x \cdot x}=\frac{1+F_{x}}{2 N}+\frac{N-1}{N} \bar{\phi}_{x},
$$

where $N$ is the number of individuals in group $x$, and $\bar{\phi}_{x}$ is the average pairwise coancestry among individuals (excluding self-coancestries).

\subsection{Mating scheme and population structure}

We suppose a single commercial population of females, maintained by mating with sires rotationally supplied from $n$ sire lines, each with the same constant size of $N_{m}$ males and $N_{f}$ females over generations. The sire lines are assumed to be completely closed to each other after the initiation of rotational mating, but with various degrees of relationships in the initial generations. Within each sire line, random mating and discrete generations are assumed. Thus, the inbreeding coefficient in each sire line at generation $t\left(F_{t}^{*}\right)$ is computed by the recurrence equation

$$
F_{t}^{*}=F_{t-1}^{*}+\frac{1}{2 N_{e}}\left(1-2 F_{t-1}^{*}+F_{t-2}^{*}\right),
$$

where $N_{e}=4 N_{m} N_{f} /\left(N_{m}+N_{f}\right)$ is the effective size of the sire line [33].

The line supplying sires to the commercial females in a given generation is referred to as the supplier at that generation. We give sequential numbers 1 , $2, \ldots, n$ to the suppliers in generations $0,1, \ldots, n-1$, respectively. Letting $S_{t-i}$ be the sequential number of the supplier in generation $t-i, S_{t-i}$ could be determined by

$$
S_{t-i}=\operatorname{MOD}(t-i, n)+1,
$$

where $\operatorname{MOD}(x, n)$ is the remainder of $x$ divided by $n$. Note that, because of the nature of rotational mating, $S_{t-i}=S_{t-i-k n}$ for a given integer number $k$. 
The groups of males and females in the sire line $S_{t-i}$ are denoted by $m\left(S_{t-i}\right)$ and $f\left(S_{t-i}\right)$, respectively. The group coancestries within and between male and female groups are assumed to be equal in a given generation $t-1$ :

$$
\bar{\phi}_{m\left(S_{t-1}\right), t-1}=\bar{\phi}_{f\left(S_{t-1}\right), t-1}=\phi_{m\left(S_{t-1}\right) \cdot f\left(S_{t-1}\right), t-1}=F_{t}^{*} .
$$

The population of commercial females is denoted by $c$. Discrete generations with the same interval as the sire lines and random mating with supplied sires were assumed in the commercial population.

\section{RECURRENCE EQUATION FOR INBREEDING COEFFICIENT OF COMMERCIAL FEMALES}

\subsection{Rotational mating with unrelated sire lines}

We first consider the case with unrelated sire lines. In this case, it is apparent that the inbreeding coefficient $\left(F_{t}\right)$ of the commercial females within the first cycle of rotation is zero; $F_{t}=0$ for $t \leq n$. In Figure 1, the pedigree diagram for $t \geq n+1$ is illustrated. Applying the operational rule of coancestry (Eq. (1)) to the diagram, we get an expression of the inbreeding of the commercial females in generation $t \geq n+1$ as

$$
\begin{aligned}
F_{t} & =\phi_{m\left(S_{t-1}\right) \cdot c, t-1} \\
& =\frac{1}{2^{n+1}}\left[\phi_{m\left(S_{t-1}\right) \cdot m\left(S_{t-1}\right)}+\phi_{f\left(S_{t-1}\right) \cdot m\left(S_{t-1}\right)}+\phi_{m\left(S_{t-1}\right) \cdot c}+\phi_{f\left(S_{t-1}\right) \cdot c}\right]_{t-n-1} .
\end{aligned}
$$

From (2) and (4), the first two group coancestries in (5) are

$$
\begin{aligned}
\phi_{m\left(S_{t-1}\right) \cdot m\left(S_{t-1}\right), t-n-1} & =\frac{1+F_{t-n-1}^{*}}{2 N_{m}}+\frac{N_{m}-1}{N_{m}} \bar{\phi}_{m\left(S_{t-1}\right), t-n-1} \\
& =\frac{1+F_{t-n-1}^{*}}{2 N_{m}}+\frac{N_{m}-1}{N_{m}} F_{t-n}^{*}
\end{aligned}
$$

and

$$
\phi_{f\left(S_{t-1}\right) \cdot m\left(S_{t-1}\right), t-n-1}=F_{t-n}^{*} .
$$

Furthermore, by noting that the supplier in generation $t$ - 1 should also be the supplier in generation $t-n-1$ (i.e. $S_{t-1}=S_{t-n-1}$ ), the last two group coancestries in (5) could be written as

$$
\phi_{m\left(S_{t-1}\right) \cdot c, t-n-1}=\phi_{f\left(S_{t-1}\right) \cdot c, t-n-1}=F_{t-n} .
$$




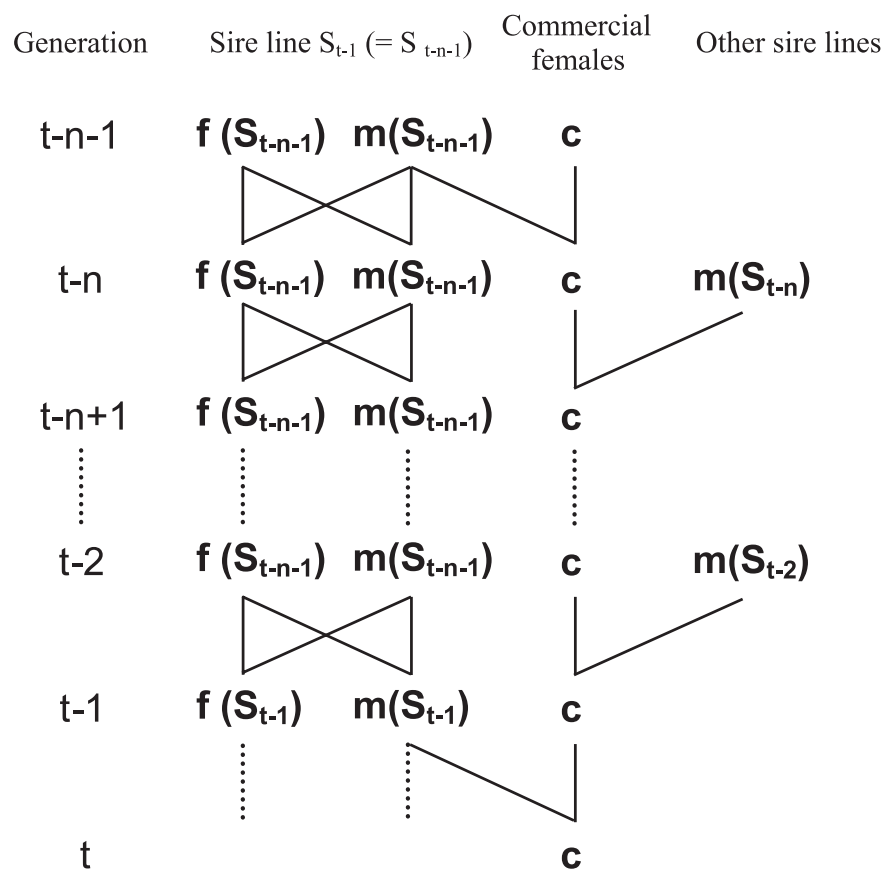

Figure 1. Rotational mating with $n$ sire lines for $t \geq n+1$. The commercial females are rotationally mated with sires supplied from male group of $n$ sire lines $(\mathrm{m}(\cdot))$. The sire line $\mathrm{S}_{t-1}$, which supplies sires for the mating at generation $t$-1, appeared as $\mathrm{S}_{t-n-1}$ in the previous cycle of mating (at generation $t-n-1$ ). Between these two generations, $n$-1 different sire lines (from $\mathrm{S}_{t-n}$ to $\mathrm{S}_{t-2}$ ) supply sires rotationally. Mating within each sire line, except for $\mathrm{S}_{t-1}$, are omitted for simplification.

Substituting these expressions into equation (5) leads to the recurrence equation for the inbreeding coefficient of the commercial females as

$$
F_{t}=\frac{1}{2^{n+1}}\left[\frac{1+F_{t-n-1}^{*}}{2 N_{m}}+\frac{2 N_{m}-1}{N_{m}} F_{t-n}^{*}+2 F_{t-n}\right] .
$$

Note that when $n=1$, the assumed mating system reduces to the closed nucleus breeding system. It can be verified that the asymptotic rate of inbreeding $\left(\Delta F=\left(F_{t}-F_{t-1}\right) /\left(1-F_{t-1}\right)\right)$ of equation (6) with $n=1$ depends only on the effective size of the sire line, and is approximated by $\Delta F=1 /\left(2 N_{e}\right)$, agreeing with the previous result for the closed nucleus breeding system $[12,13]$. 


\subsection{Rotational mating with related sire lines}

When related sire lines are used, the inbred commercial females appear within the first cycle of rotation, with the inbreeding coefficient

$$
F_{t}= \begin{cases}Q_{(2,1)} & \text { for } t=2 \\ \sum_{i=2}^{t-1} \frac{1}{2^{i-1}} R_{(t, t-i+1)}+\frac{1}{2^{t-2}} Q_{(t, 1)} & \text { for } 3 \leq t \leq n\end{cases}
$$

where

$$
Q_{(x, 1)}=\frac{1}{4}\left[\phi_{m(x) \cdot m(1)}+\phi_{f(x) \cdot m(1)}\right]_{0}
$$

and

$$
R_{(y, z)}=\frac{1}{4}\left[\phi_{m(y) \cdot m(z)}+\phi_{m(y) \cdot f(z)}+\phi_{f(y) \cdot m(z)}+\phi_{f(y) \cdot f(z)}\right]_{0}
$$

(see Appendix).

As shown in the Appendix, the inbreeding coefficient of the commercial females after the first cycle of rotation $(t \geq n+1)$ is generally expressed as

$$
F_{t}=\frac{1}{2^{n+1}}\left[\frac{1+F_{t-n-1}^{*}}{2 N_{m}}+\frac{2 N_{m}-1}{N_{m}} F_{t-n}^{*}+2 F_{t-n}\right]+\sum_{i=2}^{n} \frac{1}{2^{i-1}} R_{\left(S_{t-1}, S_{t-i}\right)} .
$$

\section{ASYMPTOTIC INBREEDING COEFFICIENT OF COMMERCIAL FEMALES}

\subsection{Rotational mating with unrelated sire lines}

When unrelated sire lines were used, the inbreeding coefficient of the commercial population eventually reaches an asymptotic value. The asymptotic value $F_{\infty}$ can be obtained by the following consideration.

Since there is no gene flow among sire lines, each line will eventually be fixed, and then

$$
F_{\infty}^{*} \equiv F_{t-n-1}^{*}=F_{t-n}^{*}=1 .
$$

Substituting this into equation (6) gives

$$
F_{t}=\frac{1}{2^{n}}\left(1+F_{t-n}\right) .
$$

Since $F_{t}$ and $F_{t-n}$ can be replaced by $F_{\infty}$ in the asymptotic state, the asymptotic value is obtained as

$$
F_{\infty}=\frac{1}{2^{n}-1}
$$




\subsection{Rotational mating with related sire lines}

With an initial relationship among sire lines, the asymptotic expression for the inbreeding coefficient of the commercial females is complicated because the second term in (8) does not converge to a single asymptotic value when $n \geq 3$. For a sufficiently large $t$, we denote the suppliers before $i$ generations as $S_{-i}$. With an analogous argument to the previous case, an asymptotic expression could be obtained as

$$
F_{\infty}=\frac{1}{2^{n}-1}\left[1+\sum_{i=2}^{n} 2^{n-i+1} R_{\left(S_{-1}, S_{-i}\right)}\right] .
$$

Equation (10) converges to a single value for $n=2$, but shows a regular oscillation with a cycle of $n$ generations for $n \geq 3$.

\section{NUMERICAL COMPUTATIONS}

\subsection{Rotational mating with unrelated sire lines}

To assess the effects of the number $(n)$ and the size $\left(N_{m}\right.$ and $\left.N_{f}\right)$ of sire lines on the accumulation of inbreeding in the commercial females $\left(F_{t}\right)$, numerical computations with (3) and (6) were carried out for the combinations of $n=$ 2, 3, 4 and 5 and $N_{m}=2,5$ and 10. Figures 2 (A), (B) and (C) show the results of $N_{m}=2,5$ and 10, respectively, under various $n$ and a fixed $N_{f}$ $(=200)$. For a given size of sire line, an increase of $n$ reduces $F_{t}$, but the effect becomes trivial when $n \geq 4$. A comparison of Figures 2 (A)-(C) reveales that although an increase of $N_{m}$ has a pronounced effect on $F_{t}$ for a relatively small $n$ (say $n \leq 3$ ), the effect is diminished as $n$ becomes larger. For example, the inbreeding coefficients of commercial females with $n=2$ reached $22.5 \%$, $12.4 \%$ and $7.1 \%$ in generations 20 for $N_{m}=2,5$ and 10, respectively, while the corresponding values with $n=5$ were $2.0 \%, 1.1 \%$ and $0.6 \%$, respectively.

As seen from (3) and (6), the number of females in each sire line $\left(N_{f}\right)$ affects the inbreeding coefficient of the commercial females only through the effective size of sire lines ( $N_{e}$ in Eq. (3)). Since the number of the less numerous sex, i.e. the number of males in this case, is the major factor for determining the effective size, it is expected that an increase of $N_{f}$ has little effect on the accumulation of inbreeding in the commercial females. For example, the inbreeding coefficient in the commercial females for the case of $N_{m}=5$ and $N_{f}=1000$ showed no essential differences from that of the case of $N_{m}=5$ and $N_{f}=200$. 

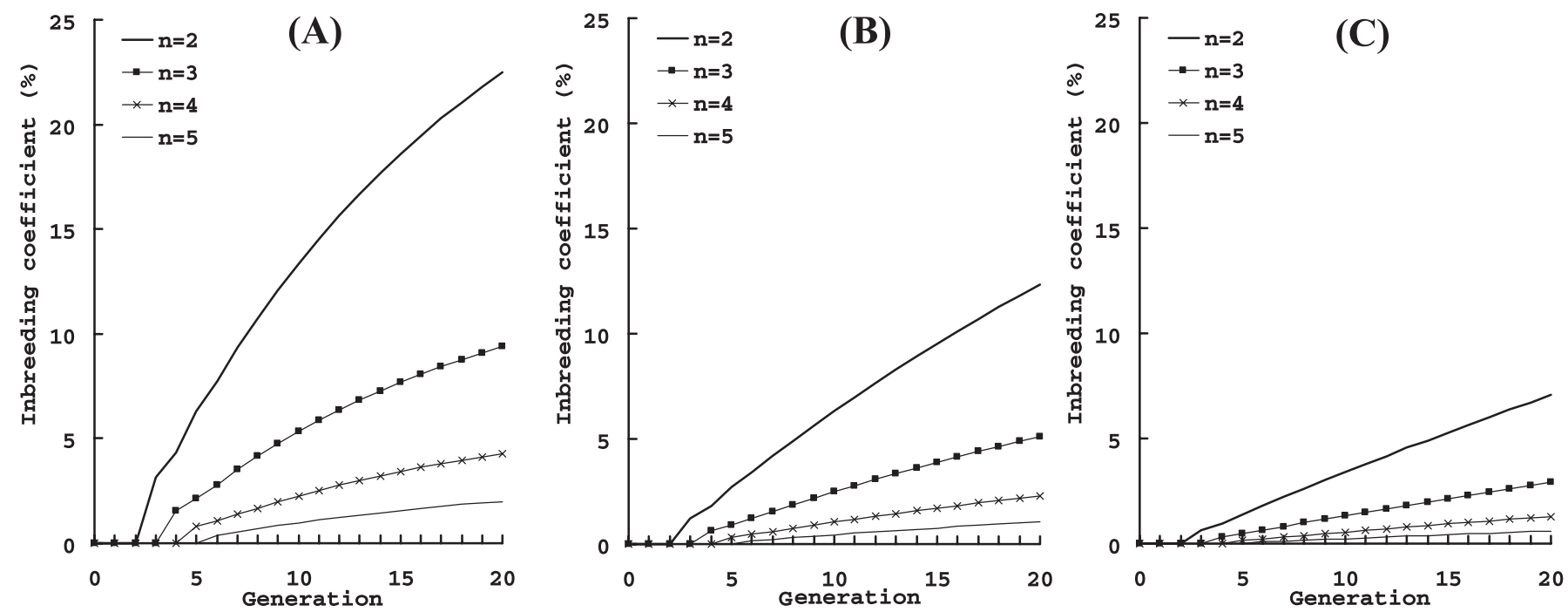

-1
7
0
0
0
0
0
0

Figure 2. Inbreeding coefficient of the commercial females under rotational mating using $n$ of unrelated sire lines, with the sizes of (A) $N_{m}=2$ males and $N_{f}=200$ females, (B) $N_{m}=5$ and $N_{f}=200$, and (C) $N_{m}=10$ and $N_{f}=200$. 


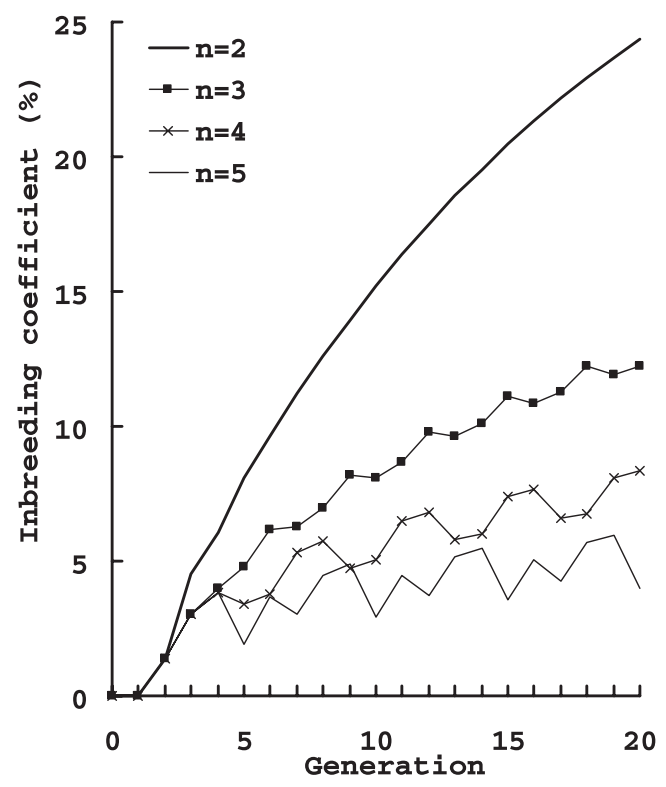

Figure 3. Inbreeding coefficient of the commercial females using $n$ of related sire lines shown in Table II, each with the size of $N_{m}=2$ males and $N_{f}=200$ females.

\subsection{Rotational mating with related sire lines}

The average coancestries among five breeding herds (i.e. Hyogo (HY), Tottori (T), Shimane (S), Okayama (O) and Hiroshima (HR) prefectures) of a Japanese beef breed (Japanese Black cattle) were used to illustrate the effect of initial relationships among sire lines. The average coancestries among the five herds estimated by Honda et al. [11] are given in Table I. We supposed a situation where a sire line with $N_{m}=2$ and $N_{f}=200$ is constructed from each of the five herds, and the rotational mating system is applied to a hypothetical population of commercial females. For the simplicity of the computation, the five sire lines were assumed to be initially inbred with the same degree of $F_{0}^{*}=0.06$, which is the average inbreeding coefficient in the current breed [11]. The orders of the use of sire lines in the commercial population were assumed to be HY-T, HY-T-S, HY-T-S-O and HY-T-S-O-HR for $n=2,3$, 4 , and 5, respectively. The inbreeding coefficient of the commercial population computed from equation (8) is shown in Figure 3.

Although the inbreeding coefficient in the commercial population is higher than the corresponding value of the case with unrelated sire lines ( $c f$. Fig. 2 (A)), the rotational mating with four or five sire lines can essentially suppress the increase of inbreeding in the commercial females. As seen from the 
Table I. The average coancestries of the Japanese Black cattle in five subpopulations of traditional breeding prefectures.

\begin{tabular}{|c|c|c|c|c|c|c|c|c|c|c|c|}
\hline \multirow[t]{2}{*}{ Subpopulation* } & \multirow[b]{2}{*}{ Sex** } & \multicolumn{2}{|c|}{$\mathrm{HY}$} & \multicolumn{2}{|c|}{$\mathrm{T}$} & \multicolumn{2}{|c|}{$\mathrm{S}$} & \multicolumn{2}{|c|}{$\mathrm{O}$} & \multicolumn{2}{|c|}{ HR } \\
\hline & & M & $\mathrm{F}$ & M & $\mathrm{F}$ & M & $\mathrm{F}$ & $\mathrm{M}$ & F & $\mathrm{M}$ & F \\
\hline \multirow{2}{*}{ HY } & $\mathrm{M}$ & - & - & & & & & & & & \\
\hline & $\mathrm{F}$ & - & - & & & & & & & & \\
\hline \multirow{2}{*}{$\mathrm{T}$} & M & 0.020 & 0.021 & - & - & & & & & & \\
\hline & $\mathrm{F}$ & 0.035 & 0.037 & - & - & & & & & & \\
\hline \multirow{2}{*}{$\mathrm{S}$} & M & 0.039 & 0.038 & 0.040 & 0.052 & - & - & & & & \\
\hline & $\mathrm{F}$ & 0.031 & 0.031 & 0.035 & 0.045 & - & - & & & & \\
\hline \multirow{2}{*}{$\mathrm{O}$} & M & 0.018 & 0.018 & 0.021 & 0.028 & 0.054 & 0.053 & - & - & & \\
\hline & $\mathrm{F}$ & 0.037 & 0.038 & 0.025 & 0.034 & 0.059 & 0.056 & - & - & & \\
\hline \multirow{2}{*}{$\mathrm{HR}$} & $\mathrm{M}$ & 0.040 & 0.038 & 0.016 & 0.021 & 0.033 & 0.027 & 0.016 & 0.021 & - & - \\
\hline & $\mathrm{F}$ & 0.040 & 0.038 & 0.015 & 0.017 & 0.024 & 0.019 & 0.011 & 0.016 & - & - \\
\hline
\end{tabular}

* Subpopulations of Hyogo (HY), Tottori (T), Shimane (S), Okayama (O), and Hiroshima (HR) prefectures.

** M: male; F: female. 
Table II. Asymptotic inbreeding coefficients (\%) of commercial females with $n$ unrelated sire lines and related sire lines.

\begin{tabular}{ccccccc}
\hline & & \multicolumn{5}{c}{ Related sire lines } \\
\cline { 3 - 7 }$n$ & Unrelated sire lines & $\mathrm{HY}$ & $\mathrm{T}$ & $\mathrm{S}$ & $\mathrm{O}$ & $\mathrm{HR}$ \\
\hline 1 & 100 & - & - & - & - & - \\
2 & 33.3 & 35.2 & 35.2 & - & - & - \\
3 & 14.3 & 17.1 & 17.1 & 17.8 & - & - \\
4 & 6.7 & 9.4 & 9.5 & 10.6 & 10.7 & - \\
5 & 3.2 & 6.6 & 5.7 & 7.1 & 7.3 & 5.2 \\
\hline
\end{tabular}

With related sire lines, inbreeding shows a regular oscillation according to coancestries among consecutive suppliers, HY, T, S, O, and HR.

additional term due to the initial relationships in equation (8), the oscillation pattern observed in the cases with $n \geq 3$ depends on the order of the use of the sire lines, and a sharp increase of inbreeding manifests when highly related sire lines are used in two consecutive generations.

\subsection{Asymptotic inbreeding coefficient in commercial females}

Asymptotic inbreeding coefficients of commercial females are presented in Table II, for the cases with unrelated and related sire lines. With unrelated sire lines, the asymptotic inbreeding coefficient of the commercial females can be suppressed to less than $10 \%$ when $n \geq 4$. Even if the sire lines are initially related, the initial relationships generate no serious increases of the asymptotic inbreeding.

\section{DISCUSSION}

There is much evidence that the inbreeding coefficients of females have substantial effects on commercially important traits, such as milk yield and egg production [20]. The traits relevant to meat production, such as carcass weight and daily gain, are also affected by the inbreeding of dams [20]. In recent years, various mating and selection strategies have been proposed to reduce the rates of inbreeding in selection programs while keeping genetic gains at a given level (e.g. $[5,15,26])$. However, these methods target small nucleus populations, in which selection and mating can be intensively managed, and may not be implemented in the commercial female populations, where the choice of sires to be mated with the females depends on the strategies of individual farmers. 
The mating system considered in the present study will have practical merit in its application to the commercial female population, because it does not require systematic controls of mating over the population, such as the systematic mating allocation required in the minimum coancestry mating [5]. The proposed system can be arranged by individual farmers according to their strategies. For example, if five sire lines (say A, B, C, D and E) are available, some farmers could rotationally use the four lines A, B, C and D, while others could adopt the mating with the four lines $\mathrm{B}, \mathrm{C}, \mathrm{D}$ and $\mathrm{E}$.

In the above context, the success of rotational mating will highly depend on the genetic merits or qualities of the available sire lines. If one sire line is inferior to the others, farmers in the commercial population will avoid the use. For successful rotational mating, well-designed breeding programs will be essential in the sire lines.

Numerical computations have shown that the critical factor for determining the inbreeding in commercial females is the number of sire lines, and the size of each sire line has a minor effect. In practice, if four or five sire lines are available, rotational mating could be an effective system to reduce the short- and long-term inbreeding of the commercial females, irrespective of the effective size of each sire line. When the sire lines are initially related, an oscillation of the inbreeding coefficient may occur in commercial females. A sharp increase in inbreeding in one generation may cause a serious inbreeding depression, as experimentally shown by Beilharz [2]. To minimize the oscillation of inbreeding, the use of two highly related lines in consecutive generations should be avoided.

Ultimately, the inbreeding coefficient of commercial females reaches an intermediate value ( $c f$. Eqs. (9) and (10)). This is a special case of the theoretical result of Robertson [22]. He showed that a population split into permanent sublines will not genetically fix, because allele fixation occurs independently in each subline. In the present study, the assumption of closed sire lines led to the fixation of the lines with different alleles, and thus the commercial females retained a heterozygosity in the ultimate state. In the study of crossbreeding, Dickerson [8] showed that the fraction of heterosis expected under rotational crossing with $n$ breeds is $\left(2^{n}-2\right) /\left(2^{n}-1\right)$. This agrees with the expected ultimate heterozygosity in the commercial females predicted by equation (9), that is $1-F_{\infty}=1-1 /\left(2^{n}-1\right)$.

The hierarchical breed structure, as assumed in the present study, has been reported in many livestock breeds (e.g. [1,23,28]). For example, in the population of Japanese Black cattle, the five regional subpopulations listed in Table I have played important roles as suppliers of breeding stocks to the entire breed. 
Although the genetic diversity of the breed has been reduced by the intensive use of sires from limited strains during the past decade [18], the five regional subpopulations still maintain their unique genetic compositions [11]. Rotational mating with the existing genetic materials will largely contribute not only to the reduction of inbreeding in the commercial females but also to the maintenance of genetic diversity in the breed through balancing of the genetic contributions of the subpopulations.

In the derivation of the theory, we have made several simplifications. Among them, the most critical one is the neglect of selection in sire lines. Selection will inflate the inbreeding coefficient in a sire line [16,21,25,31]. Due to the inflated inbreeding, the inbreeding in the commercial females will also be enhanced (cf. Eqs. (6) and (8)). This effect could be approximately taken into account by computing the effective size of sire lines with the formula incorporating the effect of selection. Theories for predicting the effective population size have been developed for various types of selection (e.g. Wray et al. [32] for index selection, and Bijma et al. [3] for BLUP selection).

In a strict sense, the application of derived equations is limited to neutral loci. If intense selection for the same breeding objective is carried out in all sire lines, it will tend to increase the genetic resemblance between sire lines, even if they are maintained separately. Thus the same favorable alleles will be fixed in most sire lines, and the heterozygosity for the loci in the commercial females may be less than that predicted.

In practice, sire lines may have different sizes. The problem of unequal sizes of sire lines could be solved straightforwardly by applying the recurrence equation (3) to each sire line. The inbreeding coefficient of the commercial females is then obtained by replacing $F_{t-n}^{*}$ and $F_{t-n-1}^{*}$ in (6) and (8) by the corresponding inbreeding coefficient of the supplier in generation $t-1$.

To apply rotational mating to species with overlapping generations, the sire lines should be rotated based on the turnover of generations in commercial females. Although the recurrence equations (6) and (8) can not be strictly applied, the inbreeding coefficient could be approximated with these equations, so long as there are no large differences among generation intervals of sire lines and commercial females. Since the asymptotic equations (9) and (10) hold exactly even in the case of overlapping generations, the upper limit of inbreeding can be estimated with these equations.

We have assumed a situation where several sire lines are available at the initiation of rotational mating. When the upper level of hierarchy has an undivided structure, it will raise a question of whether separate sire lines should be established. This would involve trade-offs between more inbreeding (and less 
genetic gain) in the individual sire lines and less inbreeding in the commercial female population. Although the exact solution should be considered in future studies, the best compromise could be empirically found by comparing various strategies for a given total size in terms of the inbreeding coefficient and genetic gain in each level of hierarchy.

Finally, although we have assumed that sire lines are closed to each other after the initiation of rotational mating, some amounts of migration should be actually allowed among the sire lines to avoid the deleterious inbreeding effect in each of them. A recent study by Wang [30] predicts that managed migration among subpopulations could maintain a substantial genetic diversity among the subpopulations, and could reduce the inbreeding rate in each subpopulation to an acceptable level. However, through migration of animals, new relationships would be generated among sire lines, and will inflate the inbreeding of the commercial females. More detailed theoretical study is necessary on this subject.

\section{ACKNOWLEDGEMENTS}

This work was partially funded by a Grant-in-Aid for Scientific Research from the Ministry of Education, Culture, Sports, Science and Technology.

\section{REFERENCES}

[1] Barker J.S.F., The breed structure and genetic analysis of the pedigree cattle breeds in Australia. I. The Jersey, Aust. J. Agric. Res. 8 (1957) 561-586.

[2] Beilharz R.G., The effect of inbreeding on reproduction in mice, Anim. Prod. 34 (1982) 49-54.

[3] Bijma P., van Arendonk J.A.M., Woolliams J.A., Predicting rates of inbreeding for livestock improvement schemes, J. Anim. Sci. 79 (2001) 840-853.

[4] Caballero A., Toro M.A., Interrelations between effective population size and other pedigree tools for management of conserved populations, Genet. Res., Camb. 75 (2000) 331-343.

[5] Caballero A., Santiago E., Toro M.A., Systems of mating to reduce inbreeding in selected populations, Anim. Sci. 62 (1996) 431-442.

[6] Cockerham C.C., Group inbreeding and coancestry, Genetics 56 (1967) 89-104.

[7] Cockerham C.C., Avoidance and rate of inbreeding, in: Kojima K. (Ed.), Mathematical Topics in Population Genetics, Springer-Verlag, New York, 1970, pp. 104-127.

[8] Dickerson G.E., Inbreeding and heterosis in animals, in: Proc. Anim. Breeding Genet. Symp. in Honor of Dr. J.L. Lush, 1973, Am. Soc. Anim. Sci., Am. Dairy Sci. Assoc., Champaign, IL, pp. 54-77. 
[9] Falconer D.S., Mackay T.F.C., Introduction to quantitative genetics, 4th edn., Longman Harlow, UK, 1996.

[10] Gowe R.S., Robertson A., Latter B.D.H., Environment and poultry breeding problems. 5. The design of poultry control strains, Poult. Sci. 38 (1959) 462-471.

[11] Honda T., Nomura T., Yamaguchi Y., Mukai F., Pedigree analysis of genetic subdivision in a population of Japanese Black cattle, Anim. Sci. J. 73 (2002) 445-452.

[12] James J.W., Open nucleus breeding systems, Anim. Prod. 24 (1977) 287-305.

[13] James J.W., Effective population size in open nucleus breeding schemes, Acta. Agric. Scand. 28 (1978) 387-392.

[14] Malécot G., The mathematics of Heredity, Freeman, San Francisco, USA, 1969.

[15] Meuwissen T.H.E., Maximizing the response of selection with a predefined rate of inbreeding, J. Anim. Sci. 75 (1997) 934-940.

[16] Nomura T., On the methods for predicting the effective size of populations under selection, Heredity 83 (1999) 485-489.

[17] Nomura T., Yonezawa K., A comparison of four systems of group mating for avoiding inbreeding, Genet. Sel. Evol. 28 (1996) 141-159.

[18] Nomura T., Honda T., Mukai F., Inbreeding and effective population size of Japanese Black cattle, J. Anim. Sci. 79 (2001) 366-370.

[19] Nozawa K., Statistical studies on the populations of farm animals, Jap. J. Breeding 10 (1960) 29-32.

[20] Pirchner F., Genetic structure of populations. 1. Closed populations or matings among related individuals, in: Chapman A.B. (Ed.), General and Quantitative Genetics, Elsevier, New York, 1985, pp. 227-250.

[21] Robertson A., Inbreeding in artificial selection programmes, Genet. Res., Camb. 2 (1961) 189-194.

[22] Robertson A., The effect of nonrandom mating within inbred lines on the rate of inbreeding, Genet. Res., Camb. 5 (1964) 164-167.

[23] Robertson A., Asker W.A., The genetic history and breed structure of British Friesian cattle, Emp. J. Exp. Agric. 19 (1951) 113-130.

[24] de Rochambeau H., Chevalet C., Minimisation des coefficients de consanguinité moyens dans les petites populations d'animaux domestiques, Génét. Sél. Évol. 17 (1985) 459-480.

[25] Santiago E., Caballero A., Effective size of populations under selection, Genetics 139 (1995) 1013-1030.

[26] Sonesson A.K., Meuwissen T.H.E., Mating schemes for optimum contribution selection with constrained rates of inbreeding, Genet. Sel. Evol. 32 (2000) 231-248.

[27] Toro M.A., Silio L., Rodrigáñez J., Rodriguez C., Fernandez J., Optimal use of genetic markers in conservation programmes, Genet. Sel. Evol. 31 (1999) $255-261$.

[28] Vu Tien Khang J., Methods of analysis of demographic and genealogical data in the populations of domestic animals, Génét. Sél. Évol. 15 (1983) 263-298.

[29] Wang J., More efficient breeding systems for controlling inbreeding and effective size in animal populations, Heredity 79 (1997) 591-599. 
[30] Wang J., Effective size and $F$-statistics of subdivided populations. II. Dioecious species, Genetics 146 (1997) 1465-1474.

[31] Wray N.R., Thompson R., Prediction of rates of inbreeding in selected populations, Genet. Res., Camb. 55 (1990) 41-54.

[32] Wray N.R., Woolliams J.A., Thompson R., Prediction of rates of inbreeding in populations undergoing index selection, Theor. Appl. Genet. 87 (1994) 878-892.

[33] Wright S., Evolution in Mendelian populations, Genetics 16 (1931) 97-159.

\section{APPENDIX}

\section{Derivation of equations (7) and (8)}

Prior to the derivation, we show a fundamental property of group coancestry among two closed lines. Figure A.1 shows two closed lines $x$ and $y$ with initial relationships $\phi_{m(x) \cdot m(y), 0}, \phi_{m(x) \cdot f(y), 0}, \phi_{f(x) \cdot m(y), 0}$ and $\phi_{f(x) \cdot f(y), 0}$. On the assumption of random mating within each line, the group coancestry between lines $x$ and $y$ at generation $t \geq 1$ is constant over generations, i.e.

$$
\phi_{x \cdot y, t}=\frac{1}{4}\left[\phi_{m(x) \cdot m(y)}+\phi_{m(x) \cdot f(y)}+\phi_{f(x) \cdot m(y)}+\phi_{f(x) \cdot f(y)}\right]_{0}=R_{(x, y)} .
$$

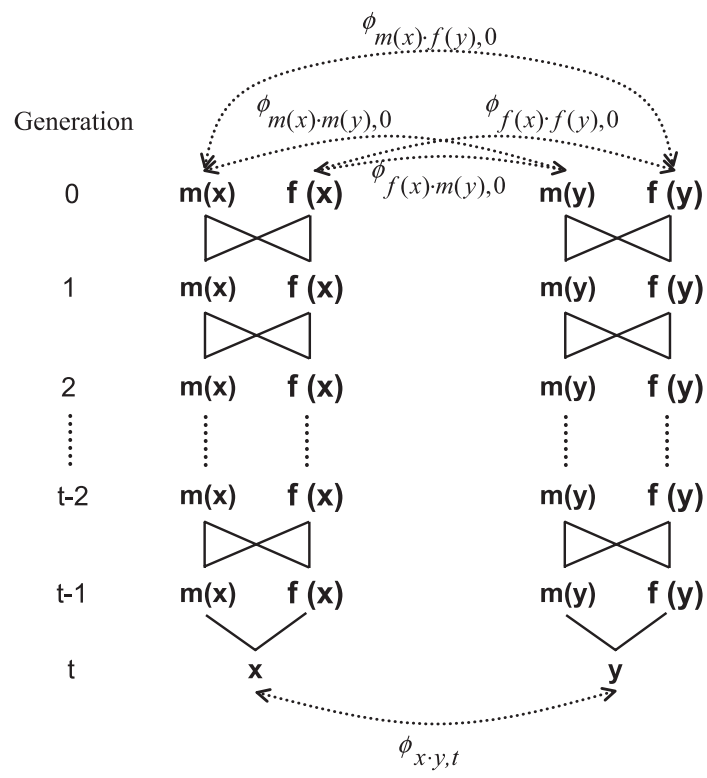

Figure A.1. Illustration for relationships between two closed sire lines $x$ and $y$ at generation $t \geq 1$, with the initial relationships $\phi_{m(x) \cdot m(y), 0}, \phi_{m(x) \cdot f(y), 0}, \phi_{f(x) \cdot m(y), 0}$ and $\phi_{f(x) \cdot f(y), 0}$. 

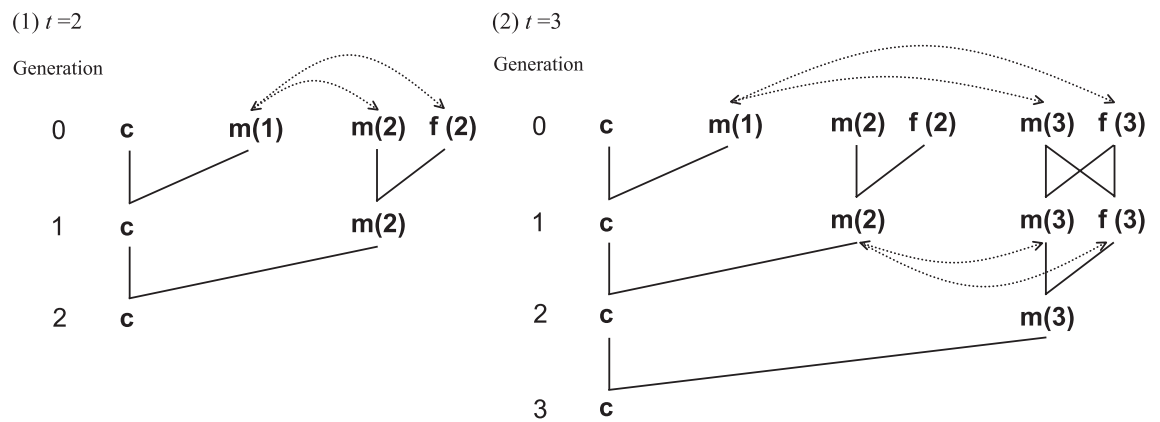

Figure A.2. Pedigree diagrams to derive the inbreeding coefficient $\left(F_{t}\right)$ of commercial females within the first cycle of rotational mating at (1) $t=2$ and (2) $t=3$ (for $n \geq 3$ ). The group coancestries relevant to $F_{t}$ are shown by dashed curves with arrows of both directions.

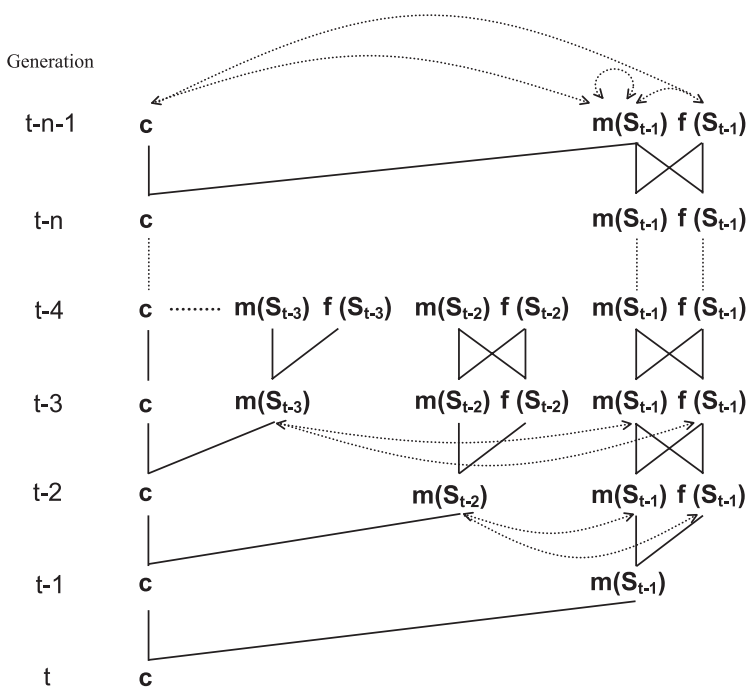

Figure A.3. Pedigree diagram to derive the inbreeding coefficient $\left(F_{t}\right)$ of commercial females after the first cycle of rotational mating $(t \geq n+1)$. The group coancestries relevant to $F_{t}$ are shown by dashed curves with arrows of both directions.

Consider first the inbreeding coefficient of commercial females within the first cycle of rotation (Eq. (7)). Applying the operational rule (Eq. (1)) to the pedigree diagram of Figure A.2 (1) leads to the inbreeding coefficient of commercial females in generation 2 as

$$
F_{2}=\phi_{m(2) \cdot c, 1}=Q_{(2,1)},
$$


where $Q_{(2,1)}$ is generally defined as $Q_{(x, 1)}=\frac{1}{4}\left[\phi_{m(x) \cdot m(1)}+\phi_{f(x) \cdot m(1)}\right]_{0}$. In Figure A.2 (2), the pedigree diagram for $t=3 \quad(n \geq 3)$ is illustrated. With the application of equations (1) and (A1) to this diagram, the inbreeding coefficient in generation 3 is obtained as

$$
\begin{aligned}
F_{3} & =\frac{1}{4}\left[\phi_{m(3) \cdot m(2)}+\phi_{f(3) \cdot m(2)}\right]_{1}+\frac{1}{8}\left[\phi_{m(3) \cdot m(1)}+\phi_{f(3) \cdot m(1)}\right]_{0} \\
& =\frac{1}{2} R_{(3,2)}+\frac{1}{2} Q_{(3,1)} .
\end{aligned}
$$

With the same argument, the inbreeding coefficient in generation $3 \leq t \leq n$ can be generally expressed as

$$
F_{t}=\sum_{i=2}^{t-1} \frac{1}{2^{i-1}} R_{(t, t-i+1)}+\frac{1}{2^{t-2}} Q_{(t, 1)} .
$$

Next, consider the inbreeding coefficient of commercial females after one cycle of rotational mating, i.e. $t \geq n+1$. The pedigree diagram is illustrated in Figure A.3. Applying the operational rule (Eq. (1)) to the pedigree diagram, we get an expression of $F_{t}$ as

$$
\begin{aligned}
F_{t}=\frac{1}{2^{n+1}}\left[\phi_{m\left(S_{t-1}\right) \cdot m\left(S_{t-1}\right)}+\phi_{f\left(S_{t-1}\right) \cdot m\left(S_{t-1}\right)}+\phi_{m\left(S_{t-1}\right) \cdot c}+\phi_{f\left(S_{t-1}\right) \cdot c}\right]_{t-n-1} \\
+\sum_{i=2}^{n} \frac{1}{2^{i}}\left[\phi_{m\left(S_{t-1}\right) \cdot m\left(S_{t-i}\right)}+\phi_{f\left(S_{t-1}\right) \cdot m\left(S_{t-i}\right)}\right]_{t-i} .
\end{aligned}
$$

With equations (6) and (A1), the above expression reduces to (8).

To access this journal online: www.edpsciences.org 